\title{
MULTIMEDIA LEARNING OBJECTS FOR DIGITAL SIGNAL PROCESSING IN COMMUNICATIONS
}

\author{
Amir Asif \\ Department of Computer Science \\ York University \\ North York, ON M3J 1P3, Canada \\ Email: asif@cs.yorku.ca
}

\begin{abstract}
Advances in the multimedia technology provide an opportunity to enhance learning in the university education. The paper presents a sample of multimedia learning objects and cooperative activities based on Macromedia Flash, Questionmark Perception, and Mathworks Matlab, used to teach the theoretical concepts in a senior level course, Digital Signal Processing in Communication Systems (DSP/CS). A study that compares the performance of students with and without multimedia access is also included.
\end{abstract}

\section{INTRODUCTION}

Interactive multimedia elements allow information to be presented in a comprehensible format, attuned to the way the students' minds work. Several courses [1]-[2] in Electrical and Computer Engineering (ECE) have used multimedia to enhance the quality of learning for the students. The paper presents a sample collection of multimedia learning objects and cooperative activities that are used to supplement the teaching material in a final year ECE course, Digital Signal Processing in Communication Systems (DSP/CS).

A multimedia learning object is defined as an animation that includes a combination of text, graphics, sound, and video packaged together. Unlike the standard lecture mode, learning objects allow flexibility and round-the-clock access to the students. Students typically run the learning objects from a compact disc (CD) or from a website. The learning objects used in DSP/CS are designed using Macromedia Flash and Questionmark Perception. Macromedia Flash allows step by step illustration of the main concepts, which the students can run at their own pace. Sample learning objects included in the paper illustrate the algorithms of Convolution, Huffman coding, Arithmetic coding, Lempel-Ziv coding, and Viterbi algorithm. Questionmark Perception is used to develop short quizzes that students write online. In addition to other statistical measures, the software provides instructors with average scores of the students on individual questions. The data is used to judge students' weaknesses, which are then addressed in the face-to-face sessions.

Hundreds of research studies on cooperative learning [3] have established that the introduction of structured, teambased activities has substantial positive effect on the performance of the students. In this paper, we describe a series of cooperative activities developed for DSP/CS. The cooperative activities are based on the student version of Matlab hence easily transportable across different computing platforms. A study that compares the performance of students with access to multimedia content versus those without access, is also included in the paper. A quantitative improvement of 10 percentage points is observed in the final scores of the students with access to the multimedia content.

The paper is organized as follows. In section 2, we present the cooperative activities. Section 3 describes the multimedia learning objects while section 4 presents the results of our comparative study on the performance of the students with and without access to the multimedia content. Finally, in section 5 we conclude the paper. In the spirit of presenting material via the web, the learning objects and cooperative activities can be accessed by visiting the web document maintained at [4] or, by using the pdf file of the paper maintained at the same web site. Words underlined in the pdf document are links to the multimedia elements.

\section{COOPERATIVE ACTIVITIES}

The objective of DSP/CS is to develop a mathematical understanding of the constituent components used in a digital communication system. Emphasis is to cover signal processing techniques used in source coding (Huffman, Arithmetic, and Dictionary Codes), signal detection in the presence of white noise, digital modulation and demodulation schemes with error performance analysis, channel coding (cyclic and convolutional codes), and spread spectrum techniques. Introduction to the information theory including the

\footnotetext{
${ }^{1}$ Matlab is a registered trademark of Mathworks.
} 


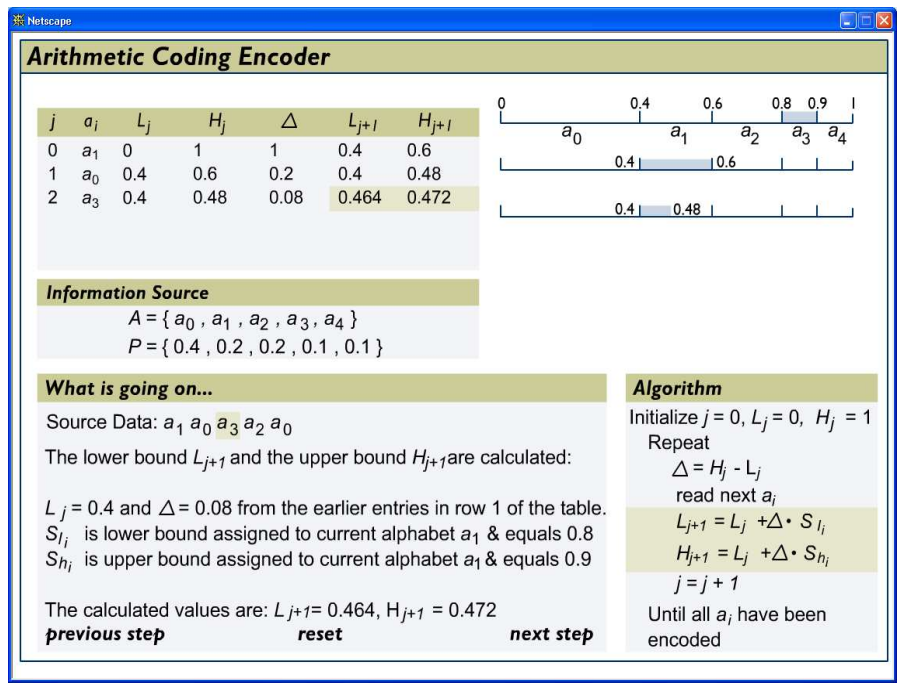

Fig. 1. Screen shot from Arithmetic encoding animation developed using Macromedia Flash.

Shannon's source coding and channel capacity bounds, is also provided at the start of the course.

The style of the course is hands-on with students having access to the multimedia learning objects, cooperative activities, and online quizzes through a course management system (CMS), [5]. The CMS is the entry to the online features of the course, developed locally with core functionality written in XML/RPC, Dynamic HTML, and Java Script. The formatting of the course on the CMS is based on the delivery model used for the course. To ensure scalability, the department developed a set [5] of course delivery models consisting of an appropriate combinations of learning methods and technologies. DSP/CS used the presentational cooperative (PC) delivery model.

The cooperative activities determine the style of the course and ensure that the students understand the underlying concepts by writing computer programs. In designing the cooperative activities, the goal has been to ensure that these are easily transportable to different platforms including workstations, personal computers, and MacIntosh's. As such, the cooperative activities are primarily based on the student version of Matlab, which is supported by most computing platforms. Oncampus students work in supervised group but for distant students, the simulations can be performed independently. The cooperative objects used in DSP/CS include:

1. Introduction of Matlab: Introduced as a review with some elementary exercises.

2. Computer Generation of Random Variables: Generate random variables with a given distribution by transforming the random variable with an uniform distribution on $(0,1)$.

3. Quadrature Amplitude Modulation (QAM): Review of continuous time analog modulation and demodulation techniques with emphasis on QAM.

4. Quantization: Simulate both uniform and nonuniform quantizers using the Lloyd-Max's algorithm.

5. Matched Filtering: Design optimum receivers for transmitting binary information through a channel corrupted with additive White Gaussian Noise (AWGN). Both signal correlator and matched filter implementations are considered and error performance analysis is performed.

6. Phase Shift Keying (PSK): Monte-Carlo simulations of $M$-ary PSK communication systems. Error performance analysis is performed by plotting the symbol error-rate versus signal to noise ratio (SNR) for AWGN.

7. Frequency Shift Keying (FSK): Same as (6) but for $M$ ary FSK communication systems. Performance is observed for different values of $M$ and compared with corresponding $M$-ary phase shift keying (MPSK).

8. PSK modulation with Convolutional Codes, [4]: MonteCarlo simulations for a binary communication system that uses convolutional encoding with PSK. Quantify improvement over PSK without convolutional codes as in (6).

9. FSK modulation with Convolutional codes: Same as (8) but with FSK modulation. Performance is compared with FSK without convolutional codes and PSK with convolutional codes as in (7) and (8).

10. Direct Sequence Spread Spectrum (DSSS) Communi-

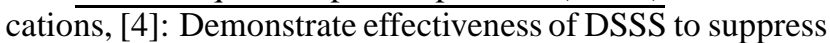
jamming interference. Error performance analysis performed. 11. Frequency Hopping Spread Spectrum (FHSS) Communications: Repeat (10) for FHSS and compare the two systems (10) and (11) in terms of symbol error-rates.

\section{LEARNING OBJECTS}

Interactive multimedia (fig. 1) enables the web presentations to be dynamic and presents information in a nonlinear format that is easier to comprehend for the learners. A series of 


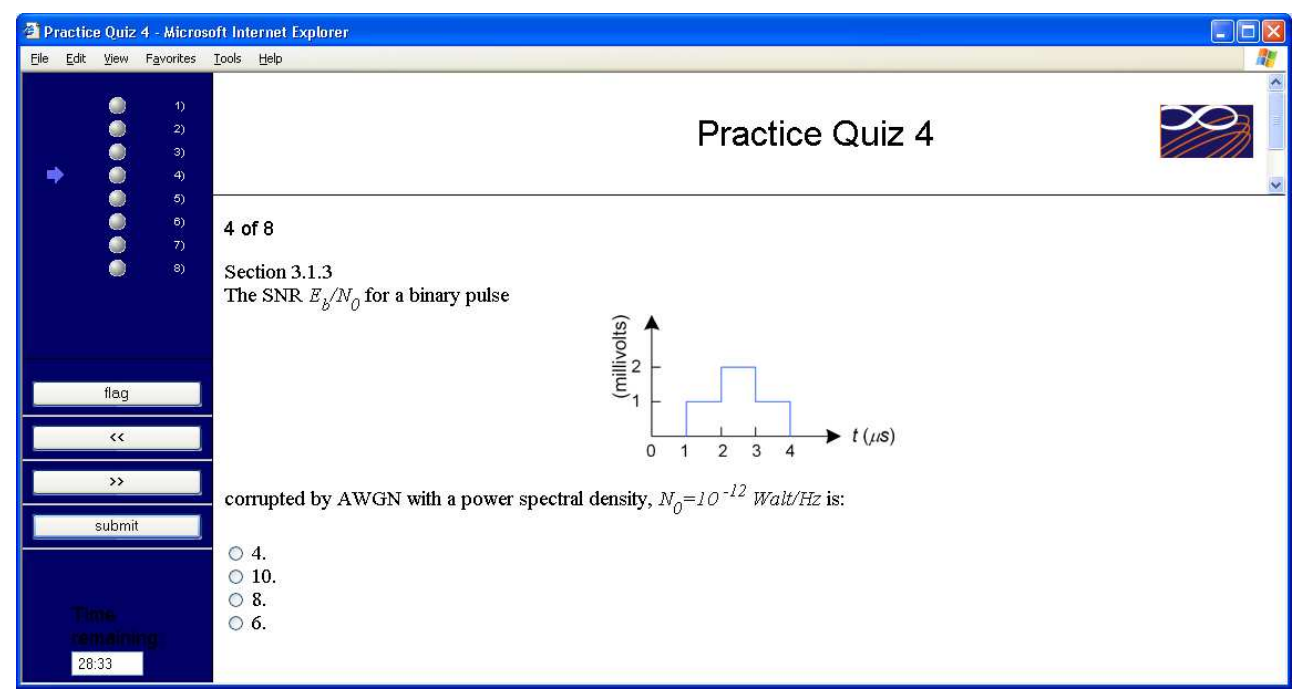

Fig. 2. A sample question from the perception Quiz.

multimedia learning objects are developed to explain the intrinsic details involved in complex algorithms. We found Macromedia Flash to be an excellent software that allowed stepwise analysis of each operation. Each animation (fig. 1) typically consists of three or four subwindows: the first subwindow details the steps involved in the algorithm, second and third subwindows use a figure and a table respectively to illustrate the results from each step, and a fourth subwindow explains the procedure followed in completing the steps. Students can run the learning objects forward or backward, one step at a time. Sample Macromedia Flash animations included in the paper are:

1. Discrete time convolution sum,

2. Continuous time convolution integral,

3. Huffman code,

4. Lempel Ziv encoder,

5. Lempel Ziv decoder,

6. Arithmetic encoder, and

7. Arithmetic decoder

These can be accessed online by visiting [4]. There is also a host of learning objects including [6] available on the web whose URLs were also made available to the learners.

Latex to HTML Formula Translator: A major hiccup in preparing technical presentations for the Web is the unavailability of an appropriate software that presents complex mathematical expressions on the web in an intelligible manner. A Latex to HTML formula translator has been developed within the Department. Mathematical expressions are first written in Latex and then translated to HTML using the software. In a few cases, some effort after translation to HTML may be required to format the spacing and other minor details in the expressions.

Perception Quizzes: After the lecture, students are encouraged to write a short quiz based on Questionmark's per- ception from their homes. The quiz facility automatically records the performance of each learner using simple numerical or multiple choice questions. The performance of the students in the perception quiz is not used for assessment but it provides the instructor with timely data on where the "sticky" spots are for the learners, which are then addressed in the coming face-to-face session. A sample question from the perception quiz is included in fig. 2. A perception session can be run by accessing the site [ 7 ].

\section{EVALUATION OF THE ONLINE EXPERIENCE}

DSP/CS was offered in the Winter semester of 2002 and the overall grades were compared with an earlier batch of students that took the course with the same instructor using the conventional lecturing approach in Winter 2001. Same outline and text were used for both offerings of the courses. Informal feedback was collected through a questionnaire.

In the lecture mode, the instructor introduced a preselected list of topics (as announced in the course outline distributed at the start of the semester) from the text and explained the important concepts in a face-to-face environment. The lectures were followed by weekly assignments consisting of some conceptual questions and a few simulations. The final grades were partially based on the performance of the students in the assignments and partially on the score received in the mid-semester and final exams. The number of students registered for the class was close to 40 .

The second offering of the course used the presentation cooperative (PC) model, which enhanced face-to-face lectures with multimedia objects and cooperative activities. The students had advance access to the course content in multimedia format as discussed above. In terms of the content of the course, the second offering was almost identical to the first offering except for the multimedia learning ob- 


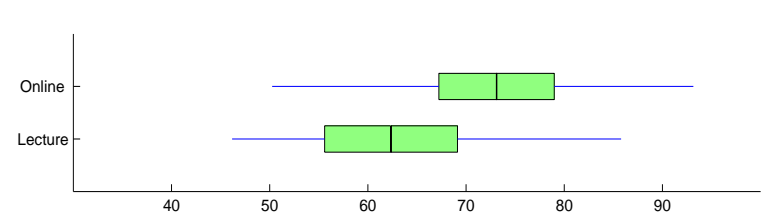

Fig. 3. Comparison of the percentage marks of the students registered in the course with: (a) access to multimedia elements versus (b) no access to multimedia elements.

jects, cooperative activities, and perception quizzes.

The final grades of the students were determined by their performance in the weekly quizzes, projects, and a final exam. The second batch of students were also assessed with a version of STAD (Student Teams-Achievement Divisions), a collaborative method developed by Slavin that is rarely used in post-secondary education. In the version used, the members of a team were given bonus marks for a test in which they all scored above a threshold. The STAD assessment was used for each of the three grading components in the second offering of DSP/CS. The scores for the second offering are normalized so that the percentage weightage of the components used for assessment stayed the same for the two offerings. Approximately 45 students registered for the course.

Qualitative feedback received from the students strongly favors the multimedia elements and cooperative activities performed in the class. In the view of the students, the multimedia content especially the learning objects and perception quizzes greatly enhanced the quality of delivery of the course. Placing material online provided round the clock accessibility and added flexibility to their schedule.

Fig. 3 illustrates the spread of the percentage score received by the students in the two sections using a box and wisker like plot. In each case, the line within the rectangle is the mean score. The rectangle shows the spread of the marks within one standard deviation (std) of the mean. For convenience of reference, we also include the mean, maximum ( $\max$ ), minimum ( $\min$ ), and standard deviation of the scores in table 1 . In terms of the mean score, there is a $10 \%$ improvement in the performance of the class with the online approach. The maximum and minimum values have also improved considerably. The improvement is attributed to collaborative learning supported by the multimedia elements.

\section{SUMMARY}

The paper describes a combination of multimedia elements including animations, perception quizzes, and cooperative activities used to supplement the teaching material in DSP/CS. The multimedia animations, referred to as learning objects, are based on Macromedia Flash and illustrate the theoreti-

\begin{tabular}{|l|c|c|c|c|}
\hline & mean & $\max$ & $\min$ & std \\
\hline Multimedia Access & 73.10 & 93.2 & 50.2 & 10.7 \\
\hline No Multimedia Access & 62.36 & 85.8 & 46.2 & 13.6 \\
\hline
\end{tabular}

Table 1. Comparison of the final score of the students with and without access to the multimedia learning objects and cooperative activities.

cal concepts and algorithms with a step by step implementation. The online quizzes are designed using Questionmark Perception that provides weekly feedback to the instructors on the understanding of the students. The cooperative activities use Mathworks Matlab and ensure that students understand the underlying concepts by writing computer programs in small groups. The performance of the students with access to multimedia elements is compared to an earlier batch, which took the same course with the same instructor but without access to the multimedia components. A quantitative improvement of $10 \%$ points is observed in the performance of the students with online access to multimedia elements.

\section{REFERENCES}

[1] J. B. Schodorf, M. A. Yoder, J. H. McClellan, and R. W. Schafer, "Using multimedia to teach the theory of digital multimedia signals," IEEE Transactions on Education, vol. 39, no. 3, pp. 336-341, August 1996.

[2] Sloan Foundation, "Catalog of online educational programs," Available http://www.sloan-c.org/catalog/.

[3] B. J. Millis and P. J. Cottell, Cooperative Learning for Higher Education Faculty, AZ: Oryx, Phoenix, 1998.

[4] A. Asif, "Sample multimedia elements for teaching digital signal processing in communications," Available http://www.cs.yorku.ca/ asif/spc/multimedia.htm.

[5] A. Asif and J. Nesbit, "Cooperative and online learning in signal processing," in Proceedings of IEEE International Conference on Acoustics, Speech, and Signal Processing, May 2001.

[6] W. J. Rugh, "Demonstrations in signals, systems, and controls," John Hopkins University (JHU), Available http://www.jhu.edu/ signals/.

[7] R. E. Slavin, Cooperative Learning: Theory, Research, and Practice, Englewood Cliffs, NJ: Prentice Hall, 1990. 